\title{
Tarifpolitische Positionen der deutschen Arbeitgeberverbände
}

Trotz der zentralen Stellung der Arbeitgeberverbände im deutschen System der industriellen Beziehungen liegen nur wenige quantitative Daten über ihre tarifpolitischen Orientierungen vor. Anhand ausgewählter Aspekte illustriert der vorliegende Beitrag erste deskriptive Befunde zur aktuellen tarifpolitischen Positionierung der Arbeitgeberverbände aus einer Befragung von Wirtschaftsverbänden 2012/2013. ${ }^{1}$ Im Zentrum stehen vor allem die Beurteilung der derzeitigen Ausgestaltung der Tarifverträge durch die Arbeitgeberverbände sowie die Haltung zu aktuell diskutierten Gesetzesinitiativen in der tarifpolitischen Arena. Die Daten beschreiben, dass die Verbände die derzeitige Ausgestaltung von Tarifverträgen tendenziell positiv bewerten, während gesetzliche Regulationen eher skeptisch betrachtet werden.

MANUEL NICKLICH

\section{Einleitung}

Nachdem um das Forschungsfeld der Tarifparteien auf Arbeitgeberseite lange Zeit eine „,auffällige Stille“ (Schmitter/Streeck 1999, S. 9) herrschte, haben Untersuchungen und Beiträge zu dem Thema in den letzten Jahren an Bedeutung gewonnen (Behrens 2011; für einen Überblick Schroeder/Weßels 2010). Quantitative Daten etwa zur tarifpolitischen Positionierung der Arbeitgeberverbände liegen hingegen wenige vor. Gleichwohl stellen die Arbeitgeberverbände wesentliche Akteure im deutschen System der industriellen Beziehungen dar, da sie im Zusammenwirken mit staatlichen Instanzen und Gewerkschaften an wesentlichen tarifpolitischen Entscheidungsprozessen maßgeblich beteiligt sind (Schroeder/Weßels 2010; Schroeder 2010). Als die Partei, die im Rahmen der Tarifautonomie zusammen mit den Gewerkschaften Flächentarifverträge abschließt, nehmen die Arbeitgeberverbände eine wesentliche Rolle bei der Entwicklung kollektiv ausgehandelter Arbeitsbedingungen in Deutschland wahr und tragen mit der Verpflichtung ihrer Mitglieder unmittelbar zu deren Umsetzung bei (u. a. Weitbrecht 2010). Betrachtet man vor diesem Hintergrund den vor allem in den letzten zwei Jahrzehnten zunehmend beobachtbaren Rückgang der Flächentarifbindung (u. a. Ellguth/Kohaut 2013), erscheinen Einschätzungen und Haltungen der Arbeitgeberverbände in Bezug auf die Gestaltung der Tarifverträge sowie einschlägiger gesetzlicher Reformvorhaben von hoher Bedeutung. Interessant ist die Frage nach der aktuellen tarifpolitischen Positionierung der Arbeitgeber vor allem dahin gehend, dass noch 2003 der ehemalige BDI-Präsident Michael Rogowski die damalige Arbeitgeberposition exemplarisch deutlich machte und festhielt: „Ich wünsche mir manchmal ein großes Lagerfeuer, um das Betriebsverfassungsgesetz und die Tarifverträge hineinzuwerfen. Danach könnte man einfach wieder von vorn anfangen.“ (Spiegel-Online vom 23.10.2003). Zu klären ist, ob sich eine derartig vehemente Ablehnung auf Arbeitgeberseite nach wie vor erkennen lässt. So ist es die Intention des vorliegenden Beitrags, Licht auf die aktuelle tarifpolitische Positionierung der deutschen Arbeitgeberverbände zu werfen, indem die Beurteilung und Haltung der Arbeitgeberverbände zur Ausgestaltung von Tarifpolitik und aktuellen tarifpolitischen Entwicklungen näher erkundet wird.

1 Die Befragung wurde im Rahmen des von der Hans-Böckler-Stiftung geförderten Projektes "Tariflosigkeit auf dem Weg zum Normalzustand? Die tarifpolitischen Folgen personalpolitischer Flexibilisierung und verbandlicher Fragmentierung" durchgeführt (Projektnr. 2011-466-2). 


\section{Datengrundlage}

Mit dem Ziel, das forschungs- und praxisrelevante Wissen über die arbeitgeberseitige Verbandsorganisation bzw. deren Verbands- und Mitgliederstrukturen sowie die politischen und wirtschaftlichen Verbandsleistungen systematisch zu erweitern, wurde 2005/2006 an der RWTH Aachen eine erste breit angelegte Befragung der Verbände durchgeführt (siehe auch Helfen 2012; Behrens/Helfen 2009; Helfen 2006 für eine detailliertere Darstellung der Ergebnisse der Befragung 2005/2006). Eine Neuauflage dieser Vorgängerstudie im Rahmen des Projekts „Tariflosigkeit auf dem Weg zum Normalzustand? Die tarifpolitischen Folgen personalpolitischer Flexibilisierung und verbandlicher Fragmentierung “ an der Freien Universität Berlin soll nun eine aktuelle Übersicht über die Entwicklungen des Verbandsgeschehens im Zeitverlauf ermöglichen und einen Einblick in die Einschätzungen und Haltungen der Verbände zu tagesaktuellen Debatten gewähren. Dementsprechend wurden neben allgemeinen Fragen zur Verbandsorganisation die aktuellen Diskurse zu tarifpolitischen Themen und gesetzlichen Reformvorhaben aufgenommen und es wurde im Rahmen einer schriftlichen Befragung von Wirtschaftsverbänden nach den Einschätzungen der verantwortlichen Geschäftsführer zu diesen Themen gefragt.

Die Grundlage der Daten bildet eine schriftliche Befragung der Geschäftsführer deutscher Wirtschaftsverbände. Aufbauend auf den Adressbeständen der Vorgängerbefragung wurde anhand verschiedener Sekundärquellen (u. a. Internetrecherchen, Verzeichnisse wie dem Bundesanzeiger 2012) eine umfangreiche Adressliste von in Deutschland tätigen Wirtschaftsverbänden erstellt, an die im Oktober 2012 in einer ersten Welle 1129 standardisierte Fragebögen verschickt wurden. Insgesamt wurden sowohl Regional-, Landes- und Bundesverbände als auch Fachgruppenverbände gleichermaßen berücksichtigt. Die Fragen bezogen sich hierbei auf die Verbandsorganisation (Domäne, Geschäftsführung, etc.), die Verbandsmitglieder (Organisationsgrade, $\mathrm{Zu}$ - und Abgänge, etc.) sowie die politischen und wirtschaftlichen Verbandsleistungen. Zusammengenommen antworteten bis zum Jahresende 2012178 Wirtschaftsverbände, von denen 121 Verbände ${ }^{2}$ als tarifpolitische Verbände sui generis behandelt werden können (Tabelle 1). Unter den antwortenden Verbänden sind 65 Arbeitgeberverbände (53,7\%), 25 Innungsverbände (20,7\%) und 31 Mischverbände, die sowohl Funktionen von Arbeitgeber- als auch von Industrieverbänden wahrnehmen (25,6 \%). Sofern diese Verbände direkt Unternehmen organisieren, haben sie im Durchschnitt 750 Mitglieder, ihr Organisationsgrad (Verhältnis der tatsächlichen zu den möglichen Mitgliedsunternehmen) in Bezug auf rechtlich selbstständige Mitgliedsunternehmen liegt nach eigenen Angaben mehrheitlich über $50 \%$. Gut $40 \%$ weisen einen Organisationsgrad unter $50 \%$ auf.

\section{TABELLE 1}

\section{Verbandsarten im Sample}

Angaben in absoluten Zahlen und in Prozent

\begin{tabular}{|c|c|c|c|}
\hline $\begin{array}{c}\text { Arbeitgeber- } \\
\text { verband }\end{array}$ & Innung & $\begin{array}{c}\text { Arbeitgeber- und } \\
\text { Industrieverband }\end{array}$ & Gesamt \\
\hline 65 & 25 & 31 & 121 \\
\hline $53,7 \%$ & $20,7 \%$ & $25,6 \%$ & $100,0 \%$ \\
\hline
\end{tabular}

Quelle: Wirtschaftsverbände in Deutschland 2012.

Mitteilunge

TABELLE 2

Verbände im Sample nach gewerkschaftlichen Organisationsbereichen

Angaben in absoluten Zahlen und in Prozent

\begin{tabular}{|l|c|c|c|c|c|c|}
\hline Welle & IG Metall & Ver.di & IG BCE & IG BAU & Sonstige & Gesamt \\
\hline $2012 / 2013$ & 25 & 37 & 11 & 25 & 23 & 121 \\
\hline & $20,7 \%$ & $30,6 \%$ & $9,1 \%$ & $20,7 \%$ & $19,0 \%$ & $100,0 \%$ \\
\hline
\end{tabular}

Quelle: Wirtschaftsverbände in Deutschland 2012.

Gemäß der branchenbezogenen Verbandsdomäne wurde eine Zuordnung zu den entsprechenden gewerkschaftlichen Organisationsbereichen vorgenommen, wie aus $T a$ belle 2 ersichtlich wird.

Ein gutes Fünftel ist dem Organisationsbereich der Industriegewerkschaft Metall (IG Metall) zuzuordnen und vertritt u. a. die Metallindustrie, die Elektroindustrie und den Maschinen- und Anlagenbau. Dem klassischen Dienstleistungsbereich und damit der Vereinten Dienstleistungsgewerkschaft (ver.di) sind gut $30 \%$ der antwortenden Verbände zuzuordnen, u. a. die Bankenbranche, das Gesundheitswesen sowie der Einzelhandel. Verbände, die den Branchen Energie und Chemie und damit dem Organisationsbereich der Industriegewerkschaft Bergbau Chemie Energie (IG BCE) zugeordnet werden können, sind mit etwas weniger als $10 \%$ vertreten. Das Baugewerbe, Garten- und Landschaftsbau sowie die Gebäudereinigung, die dem gewerkschaftlichen Organisationsbereich der Industriegewerkschaft Bauen Agrar Umwelt (IG BAU) zugerechnet werden, machen ein weiteres Fünftel des vorliegenden Samples aus. Das letzte Fünftel verteilt sich auf den Bereich Sonstige, zu dem unter anderem der gewerkschaftliche Organisationsbereich der Gewerkschaft Nahrung, Ge-

2 Da sich der Text in erster Linie mit der tarifpolitischen Positionierung von Arbeitgeberverbänden befasst, wird die Auswertung dementsprechend auf diese 121 Arbeitgeberverbände beschränkt. 
TABELLE 3

\section{Einschätzungen von Arbeitgeberverbänden zur Ausdifferenzierung von Tarifverträgen}

Angaben in absoluten Zahlen und in Prozent

\begin{tabular}{|c|c|c|c|}
\hline $\begin{array}{l}\text { Verbände nach } \\
\text { gewerkschaftlichem } \\
\text { Organisationsbereich }\end{array}$ & $\begin{array}{c}\text { Ablehnung } \\
\text { in } \%\end{array}$ & $\begin{array}{l}\text { Zustimmung } \\
\text { in } \%\end{array}$ & $\mathbf{n}$ \\
\hline & \multicolumn{3}{|c|}{ zu Aussage Die Tarifverträge decken zu viele Einzelaspekte ab: } \\
\hline IG Metall & 81,8 & 18,2 & 22 \\
\hline Ver.di & 53,1 & 46,9 & 32 \\
\hline IG BCE & 100,0 & 0,0 & 11 \\
\hline IG BAU & 82,6 & 17,4 & 23 \\
\hline Sonstige & 73,9 & 26,1 & 23 \\
\hline Gesamt & 73,9 & 26,1 & 111 \\
\hline \multicolumn{4}{|c|}{ Pearson $\chi 2(4) 12,6568^{* *}$} \\
\hline & \multicolumn{3}{|c|}{ zu Aussage Tarifverträge bieten ausreichend Gestaltungsspielräume für die einzelnen Unternehmen: } \\
\hline IG Metall & 37,5 & 62,5 & 24 \\
\hline Ver.di & 56,3 & 43,7 & 32 \\
\hline IG BCE & 18,2 & 81,8 & 11 \\
\hline IG BAU & 26,1 & 73,9 & 23 \\
\hline Sonstige & 60,9 & 39,1 & 23 \\
\hline Gesamt & 43,4 & 56,6 & 113 \\
\hline \multicolumn{4}{|c|}{ Pearson $\chi 2(4) 11,0051 * *$} \\
\hline
\end{tabular}

\begin{tabular}{|c|c|c|c|}
\hline \multicolumn{4}{|c|}{ Pearson $\chi 2(4) 11,0051 * *$} \\
\hline \multirow[b]{2}{*}{ IG Metall } & \multicolumn{3}{|c|}{ zu Aussage Die Tarifverträge sind zu wenig nach Unternehmensgrößen differenziert: } \\
\hline & 62,5 & 37,5 & 24 \\
\hline Ver.di & 58,1 & 41,9 & 31 \\
\hline IG BCE & 81,8 & 18,2 & 11 \\
\hline IG BAU & 72,7 & 27,3 & 22 \\
\hline Sonstige & 52,2 & 47,8 & 23 \\
\hline \multirow[t]{3}{*}{ Gesamt } & 63,1 & 36,9 & 111 \\
\hline & \multicolumn{2}{|c|}{ Pearson $\chi 2(4) 4,0498$} & \\
\hline & \multicolumn{3}{|c|}{ zu Aussage Die Tarifverträge sind ausreichend nach Regionen differenziert: } \\
\hline IG Metall & 56,5 & 43,5 & 23 \\
\hline Ver.di & 54,8 & 45,2 & 31 \\
\hline IG BCE & 36,4 & 63,6 & 11 \\
\hline IG BAU & 43,5 & 56,5 & 23 \\
\hline Sonstige & 78,3 & 21,7 & 23 \\
\hline \multirow[t]{2}{*}{ Gesamt } & 55,9 & 44,1 & 111 \\
\hline & \multicolumn{2}{|c|}{ Pearson $\chi 2(4) 7,8237^{*}$} & \\
\hline
\end{tabular}

Quelle: Wirtschaftsverbände in Deutschland 2012.

nuss, Gaststätten (NGG), d.h. insbesondere die Nahrungsmittel- und Gastronomiebranche, gezählt wird.

\section{Beurteilung der Tarifverträge}

Hinsichtlich der hier relevanten Aspekte zur Beurteilung der Tarifverträge, die vor allem vor dem Hintergrund der tarifvertraglichen Erosionstendenzen und der zentralen Rolle der Verbände von Bedeutung ist, sowie der aktuellen tarifpolitischen Entwicklung in Bezug auf gesetzgeberische Reformpläne wurden die Geschäftsführer der befragten Verbände gebeten, Einschätzungen bezüglich der Items auf einer 5-stufigen Likert-Skala vorzunehmen. Diese reichte von „trifft überhaupt nicht zu“ (1) bis „trifft voll und ganz zu“ (5). Die tarifpolitische Positionierung der Arbeitgeberverbände wurde dabei in zwei Blöcken abgefragt, die je vier Aussagen beinhalteten, zu denen Stellung bezogen werden sollte. Mit Blick auf die Ausgestaltung der Tarifverträge wurde die Differenzierung wie folgt operationalisiert:

- durch die Frage nach zu vielen Einzelaspekten, - nach hinreichend Gestaltungsspielräumen 
TABELLE 4

Einschätzungen von Arbeitgeberverbänden zu gesetzlichen Reformvorhaben tarifpolitischer Aspekte

Angaben in absoluten Zahlen und in Prozent

\begin{tabular}{l|c|c}
\hline $\begin{array}{l}\text { Verbände nach } \\
\text { gewerkschaftlichem } \\
\text { Organisationsbereich }\end{array}$ & $\begin{array}{c}\text { Ablehnung } \\
\text { in } \%\end{array}$ & $\begin{array}{c}\text { Zustimmung } \\
\text { in \% }\end{array}$ \\
\hline
\end{tabular}

zu Aussage Gesetzliche Mindestlöhne sind ein sinnvolles Instrument, um einen fairen Wettbewerb auf dem Arbeitsmarkt zu sichern:

\begin{tabular}{l|c|c|c|} 
IG Metall & 87,5 & 12,5 & 24 \\
Ver.di & 78,1 & 21,9 & 32 \\
IG BCE & 90,0 & 10,0 & 10 \\
IG BAU & 56,5 & 43,5 & 23 \\
Sonstige & 90,9 & 9,1 & 22 \\
Gesamt & $79,3 \quad 20,7$ & 111 \\
& Pearson $\chi 2(4) 10,7756^{* *}$ & \\
\hline
\end{tabular}

zu Aussage Der Abnahme derTarifbindung ist dadurch zu begegnen, dass Tarifverträge einfacher für allgemeinverbindlich erklärt werden können:

\begin{tabular}{l|c|c|c|}
\hline IG Metall & 75,0 & 25,0 & 24 \\
Ver.di & 87,1 & 12,9 & 31 \\
IG BCE & 70,0 & 30,0 & 10 \\
IG BAU & 47,6 & 52,4 & 21 \\
Sonstige & 86,4 & 13,6 & 22 \\
Gesamt & $75,0 \quad 25,0$ & 108 \\
& Pearson $\chi 2(4) 12,4647^{* *}$ & \\
\hline
\end{tabular}

zu Aussage Die Zunahme von Werkverträgen erfordert eine gesetzliche Regulierung, um eine schleichende Tarifflucht zu unterbinden:

\begin{tabular}{|c|c|c|c|}
\hline IG Metall & 87,0 & 13,0 & 23 \\
\hline Ver.di & 90,3 & 9,7 & 31 \\
\hline IG BCE & 90,0 & 10,0 & 10 \\
\hline IG BAU & 61,9 & 38,1 & 21 \\
\hline Sonstige & 95,2 & 4,8 & 21 \\
\hline Gesamt & 84,9 & 15,1 & 106 \\
\hline \multicolumn{4}{|c|}{ Pearson $\chi 2(4) 11,4059 * *$} \\
\hline
\end{tabular}

zu Aussage Eine gesetzliche Stärkung derTarifeinheitlichkeit kann der Zersplitterung der Tarifparteien entgegenwirken:

\begin{tabular}{l|c|c|c|}
\hline IG Metall & 47,8 & 52,2 & 23 \\
\hline Ver.di & 35,5 & 64,5 & 31 \\
\hline IG BCE & 10,0 & 90,0 & 10 \\
IG BAU & 40,9 & 59,1 & 22 \\
Sonstige & 42,9 & 57,1 & 21 \\
Gesamt & 38,3 & 61,7 & 107 \\
& & Pearson $\chi 2(4) 4,6235$ & \\
\hline
\end{tabular}

Quelle: Wirtschaftsverbände in Deutschland 2012.

- sowie durch die Berücksichtigung der Unternehmensgröße und Region.

Im Folgenden wird bei der Darstellung der Ergebnisse neben der Zahl der antwortenden Verbände und deren Zustimmung respektive Ablehnung in Bezug auf die einzelnen Fragen auch eine Differenzierung nach gewerkschaftlichen Organisationsbereichen vorgenommen. Damit kann nicht nur die allgemeine Tendenz tarifpolitischer Positionierung der Arbeitgeberverbände abgebildet, sondern es können gegebenenfalls auch branchenspezifische Unterschiede identifiziert werden. Die Kategorie Zustimmung erfasst dabei in konservativer Zusammenfassung ausschließlich die Ausprägungen „trifft eher zu“ (4) und „trifft voll und ganz

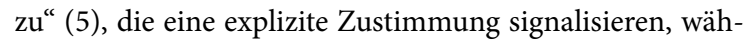
rend unter Ablehnung die Ausprägungen „trifft über- 
haupt nicht zu“ (1), „trifft eher nicht zu“ (2) und „teils/teils“ (3) subsumiert werden.

Blickt man auf den ersten Block, der sich mit den Einschätzungen zur Ausgestaltung beziehungsweise Ausdifferenzierung der Tarifverträge beschäftigt, ergibt sich allgemein betrachtet eine insgesamt deutlich positive Einschätzung der Tarifverträge durch die Verbandsvertreter. So werden die Tarifverträge mit Ausnahme des Aspektes einer ausreichenden regionalen Differenzierung mehrheitlich als hinreichend ausdifferenziert wahrgenommen (Tabelle 3).

Im Einzelnen wird der Aussage „Die Tarifverträge decken zu viele Einzelaspekte ab“ nur von einem Viertel $(26,1 \%)$ der antwortenden Verbände zugestimmt, während drei Viertel $(73,9 \%)$ dieses Statement ablehnen. Hohe Ablehnungsanteile sind dabei insbesondere im Organisationsbereich von IG Metall (81,8 \%), IG BCE (100\%) und IG BAU (82,6 \%) zu erkennen, während der Dienstleistungsbereich am deutlichsten von den restlichen Einschätzungen zu Einzelaspekten in Tarifverträgen abweicht.

Die Einschätzungen der Geschäftsführer zur Aussage „Tarifverträge bieten ausreichend Gestaltungsspielräume für die einzelnen Unternehmen“ bietet ein weniger deutliches Bild als das erste Item; sie wird allerdings immer noch mehrheitlich (56,6 \%) als positiv bewertet und damit der Gestaltungsspielraum für die betroffenen Unternehmen als ausreichend erachtet. Die größte Zustimmung erfährt die Aussage von Verbänden aus den gewerkschaftlichen Organisationsbereichen der IG BCE $(81,8 \%)$ und der IG BAU $(73,9 \%)$, während in den Organisationsbereichen von Ver. di $(43,7 \%)$ und den sonstigen Gewerkschaften $(39,1 \%)$ eine Minderheit der Verbände die Aussage für zustimmungsfähig hält und die Gestaltungspielräume der Unternehmen im Rahmen der Tarifverträge als nicht ausreichend erachtet.

Mit einem Anteil von 63,1 \% lehnen ungefähr zwei Drittel der Verbandsvertreter die Aussage „Die Tarifverträge sind zu wenig nach Unternehmensgrößen differenziert" $a b$ und schätzen damit die tarifpolitische Ausdifferenzierung nach Unternehmensgröße als hinreichend ein. Der größte Anteil an Verbänden (81,8\%), die diese Aussage als nicht zutreffend empfinden, findet sich im gewerkschaftlichen Organisationsbereich der IG BCE.

Die Aussage „Die Tarifverträge sind ausreichend nach Regionen differenziert" weicht im Ergebnis ein wenig von den anderen Resultaten ab. So wird die Aussage insgesamt von 55,9\% der Verbände als nicht zutreffend eingeschätzt, während nur 44,1 \% der Aussage zustimmen. Lediglich Verbände aus dem gewerkschaftlichen Organisationsbereich der IG BCE und der IG BAU bewerten die Aussage mit einem Anteil von 63,6 beziehungsweise 56,5\% zustimmend. Die Verbände aus den anderen gewerkschaftlichen Organisationsbereichen hingegen halten die Ausdifferenzierung nach Regionen mehrheitlich für nicht ausreichend, wobei sich insbesondere die Verbände des sonstigen gewerkschaftlichen Organisationsbereichs mit einem Ablehnungsanteil von $78,3 \%$ hervorheben.
Auffällig - aufgrund der starken sozialpartnerschaftlichen Tradition in diesem Bereich (vgl. Helfen in diesem Heft) allerdings wenig überraschend - bei der Betrachtung der Ergebnisse zu den Einschätzungen im Hinblick auf die Ausdifferenzierung der Tarifverträge ist, dass man insbesondere bei den Verbänden im gewerkschaftlichen Organisationsbereich der IG BCE durchweg die positivsten Werte - auch betreffend der Ausdifferenzierung nach Regionen bezüglich der Ausgestaltung der Tarifverträge verzeichnen kann; die Verbände aus diesem Bereich also als diejenigen, die am zufriedensten mit der Ausgestaltung der Tarifverträge sind, interpretiert werden können.

\section{Aktuelle gesetzliche Regelungs- vorhaben}

Etwas anders stellen sich die Einschätzungen der geschäftsführenden Verbandsrepräsentanten zum Thema gesetzliche Reformaktivitäten und -vorhaben dar. Zur Einschätzung aktuell diskutierter Themen gesetzlicher Regulierung tarifpolitischer Aspekte wurden Aussagen zu Mindestlöhnen, der Vereinfachung der Allgemeinverbindlicherklärung von Tarifverträgen, Einschränkung des Einsatzes von Werkverträgen sowie der Stärkung der Tarifeinheit herangezogen. Mit Ausnahme der gesetzlichen Stärkung der Tarifeinheit, die mehrheitlich befürwortet wird, werden gesetzliche Regulierungen mit großer Mehrheit negativ bewertet und abgelehnt, wie Tabelle 4 illustriert.

Die Aussage „Gesetzliche Mindestlöhne sind ein sinnvolles Instrument, um einen fairen Wettbewerb auf dem Arbeitsmarkt zu sichern" wird mit einer großen Mehrheit von 79,3\% der antwortenden Verbände abgelehnt, während lediglich 20,7 \% dieses Statement befürworten. Interessant ist insbesondere die Tatsache, dass die Verbände aus dem gewerkschaftlichen Organisationsbereich der IG BAU die Aussage mit 56,5 \% weit weniger ablehnen als die Verbände aus den restlichen Organisationsbereichen. Das ist insofern bemerkenswert, als es sich dabei um Verbände handelt, die schon länger mit dem Instrument (etwa in der Gebäudereinigung) konfrontiert sind. Ihre Erfahrung damit kann wohl als nicht unbedingt schlecht bewertet werden.

Mit einem Anteil von drei Vierteln der Verbände (75,0 \%), die die Aussage „Der Abnahme der Tarifbindung ist dadurch zu begegnen, dass Tarifverträge einfacher für allgemeinverbindlich erklärt werden können“ ablehnen, ist die Zahl zwar etwas geringer als die Ablehnung der gesetzlichen Regulierung durch Mindestlöhne, aber immer noch verhältnismäßig groß. Auch im Falle dieser Frage ist festzustellen, dass die Ablehnung bei Verbänden aus dem gewerkschaftlichen Organisationsbereich der IG BAU wesentlich geringer ausfällt $(47,6 \%)$ als bei den Verbänden der anderen Organisationsbereiche, die mit mindestens 70,0 \% diese Einschätzung ablehnen. Und auch hier kann einer 
ähnlichen Deutung wie bei dem vorhergehenden Aspekt der Mindestlöhne gefolgt und auf die schon vorhandene Erfahrung der Verbände aus diesem Bereich mit allgemeinverbindlich erklärten Tarifverträgen verwiesen werden.

Die größte Ablehnung durch die Arbeitgeberverbände erfährt allerdings die Aussage „Die Zunahme von Werkverträgen erfordert eine gesetzliche Regulierung, um eine schleichende Tarifflucht zu unterbinden“. So stimmen lediglich $15,1 \%$ der Verbände aus dem Sample diesem Satz zu, während $84,9 \%$ ihn als nicht zutreffend erachten. Hervorzuheben ist hierbei die Ablehnung der Verbände aus den Organisationsbereichen der sonstigen Gewerkschaften, die mit 95,2 \% weit über der durchschnittlichen Ablehnungsquote liegen - wobei diese auch bei den Verbänden aus den gewerkschaftlichen Organisationsbereichen von IG Metall $(87,0 \%)$, ver.di $(90,3 \%)$ sowie IG BCE $(90,0 \%)$ bemerkenswert hoch ist. Im gewerkschaftlichen Organisationsbereich der IG BAU hingegen ist die Ablehnung wiederum geringer (61,9\%), obgleich sie auch hier bei fast zwei Drittel der antwortenden Verbände liegt. Es wird demnach deutlich, dass die Verbände insbesondere in diesem Bereich keinen Handlungsbedarf und speziell keine Kausalität zur Tarifflucht sehen.

Eine Ausnahme bei den Einschätzungen zur gesetzlichen Regulation tarifpolitischer Aspekte bildet die Bewertung zur Aussage „Eine gesetzliche Stärkung der Tarifeinheitlichkeit kann der Zersplitterung der Tarifparteien entgegenwirken“. Diese wird lediglich mit einem Anteil von 38,3 \% abgelehnt, während eine Mehrheit von 61,7 \% der Verbände gesetzliche Eingriffe in diesem Sinne befürworten. Am stärksten befürworten hierbei die Verbände aus dem gewerkschaftlichen Organisationsbereich der IG BCE diese Aussage (90,0 \%), während insbesondere im Metallbereich die Einschätzungen wesentlich verhaltener ausfallen und nur knapp die Hälfte der Verbände (52,2 \%) eine gesetzliche Regulierung als positiv bewertet. In diesem Fall kann die mehrheitliche Befürwortung einer gesetzlichen Regulierung als eine Vermeidungsstrategie gegenüber einer Multiplikation von Konfliktfeldern gedeutet werden.

Im Hinblick auf die Einschätzungen gesetzlicher Regulierungen durch die Geschäftsführer ist speziell in Bezug auf die Einführung von Mindestlöhnen, die Erleichterung von Allgemeinverbindlicherklärungen sowie die Einschränkungen des Einsatzes von Werkverträgen die weitaus geringere Ablehnung durch Verbände aus dem gewerkschaftlichen Organisationsbereich der IG BAU hervorzuheben. Obwohl alle Formen gesetzlicher Regulation tarifpolitischer Aspekte abgelehnt werden, erfährt die aktuell viel diskutierte Regulation von Werkverträgen die weitaus größte Ablehnung.

\section{Diskussion}

Angesichts der äußerst spärlichen Datenlage der Verbandsorganisation in Deutschland bietet die durchgeführte Er- hebung eine Chance, Analysen zum Verbandsgeschehen zu erweitern. Auch wenn dieser Beitrag nur erste deskriptive Auswertungen der Befragung 2012/2013 darstellt, lassen die Befunde bezüglich der tarifpolitischen Positionierung der deutschen Arbeitgeberverbände erkennen, dass die Geschäftsführer der Verbände die Ausgestaltung der Tarifverträge tendenziell eher positiv bewerten, während man gesetzlichen Regulierungen tarifpolitischer Aspekte - mit Ausnahme der gesetzlichen Stärkung der Tarifeinheit - im Allgemeinen eher skeptisch bis ablehnend gegenübersteht. So scheinen sich die Arbeitgeberverbände nach der ungefähr von 2000 bis 2005 andauernden heißen Phase von Deregulierungsforderungen und -maßnahmen in Bezug auf den Flächentarifvertrag (wie etwa die Forderung nach gesetzlichen Öffnungsklauseln) (exemplarisch Schnabel 2003) (wieder) mit Sozialpartnerschaft und dem Instrumentarium von Tarifverträgen arrangiert zu haben, sodass insbesondere die Ausdifferenzierung der Flächentarifverträge durchaus positiv bewertet wird, wenn auch je nach gewerkschaftlichem Organisationsbereich in unterschiedlichem Ausmaß. Die aktuell diskutierten Gesetzesvorschläge werden von den Verbänden hingegen überwiegend abgelehnt. So werden Instrumente der direkten Lohnregulierung, wie gesetzlicher Mindestlohn, Außenseitererstreckung (AVE) sowie eine gesetzliche Regulierung der Werkvertragsvergabe, deutlich negativ beurteilt, auch wenn in Bezug auf den Grad der Ablehnung Unterschiede zwischen den Branchen zu erkennen sind. Gesetzliche Eingriffe zur Stärkung der Tarifeinheit werden jedoch eindeutig befürwortet.

Die hier vorgestellten Daten eröffnen einen seltenen Blick auf die tarifpolitische Positionierung der Arbeitgeberverbände, sind jedoch mit weitergehenden Analysen zu untermauern. Dennoch können in der Zusammenschau einige plausible Interpretationen der Befunde angeboten werden, die als Grundlage einer solchen Analyse dienen können. So kann als eine mögliche Erklärung für die positive Einschätzung tarifpolitischer Instrumente die Erfahrung mit Gewerkschaftsreaktionen in der Krise (2008/2009) herangezogen werden, die insgesamt das Ansehen von Sozialpartnerschaft erhöht und in diesem Zusammenhang auch das Vertrauen in das Gegenüber sowie die Instrumente des Systems industrieller Beziehungen gestärkt hat (zur veränderten sozialpartnerschaftlichen Orientierung der Arbeitgeberverbände siehe Helfen in dieser Ausgabe). Gleichzeitig sind viele Forderungen der Arbeitgeberseite zur Flexibilisierung des Flächentarifsystems durchgesetzt worden, was speziell in der Zufriedenheit und der positiven Bewertung der tarifvertraglichen Ausdifferenzierung durch die Geschäftsführer der Arbeitgeberverbände Ausdruck finden mag.

Eine ebenso relevante Rolle kann allerdings auch den viel diskutierten gesetzlichen Regulierungsvorschlägen (bspw. Mindestlohn) zugesprochen werden. So lassen diese sozialpartnerschaftliche Lösungen wie tarifvertragliche Entgeltgestaltung möglicherweise akzeptabler erscheinen. Der noch amtierende Präsident der Bundesvereinigung der 
Deutschen Arbeitgeberverbände Dieter Hundt hob beispielsweise jüngst die Bedeutung tarifautonom geschlossener Abkommen hervor (BDA 2013). Er verbalisierte die vermeintliche Bedrohung durch gesetzliche Regulierungen schon zuvor, indem er konstatierte, dass durch staatliche Eingriffe „die Konsequenzen gesellschaftlicher Entwicklungen alleine auf die Unternehmen ab[ge]wälzt [werden] “ (Südwestmetall 2012). Anzumerken ist in diesem Kontext allerdings die Tatsache, dass insbesondere die Geschäftsführer der Verbände, die schon Erfahrung mit gesetzlichen Regelungen gemacht haben, wie etwa Verbände aus dem gewerkschaftlichen Organisationsbereich der IG BAU, die Gesetzesvorhaben weit weniger negativ bewerten und ihre Erfahrungen mit den Instrumenten vielmehr als positiv gedeutet werden können. Die Befürwortung der gesetzlichen Regelung zur Tarifeinheit durch die Arbeitgeberverbände hingegen kann möglicherweise mit den Erfahrungen mit den zunehmend aufkommenden Berufsgewerkschaften (Piloten, Flugbegleiter, Lokomotivführer etc.) zusammenhängen und mit den oft medienwirksamen tarifpolitischen Auseinandersetzungen dieser häufig durchsetzungsstarken Interessenorganisationen. Diese potenzielle Multiplikation möglicher Konfliktherde lässt die Tarifeinheit und deren gesetzliche Durchsetzung offenbar als attraktive Alternativen für die Arbeitgeberverbände erscheinen. Wobei anzumerken ist, dass die entstehende Tarifpluralität und etwaige Segmentationstendenzen oft auch als durch Reorganisation initiierte (Outsourcing, Übernahmen etc. über Branchengrenzen hinweg) und damit hausgemachte Probleme zu interpretieren sind.

\section{LITERATUR}

Bundesvereinigung der Arbeitgeberverbände (BDA) (2013): Mindestlohn-Pläne von SPD und Grünen gefährden Beschäftigung und Tarifautonomie, Presse-Information 042/2013, http://www.bda-online.de/www/arbeitgeber.nsf/res/ PI04213.pdf/\$file/PI04213.pdf

Behrens, M. (2011): Das Paradox der Arbeitgeberverbände. Von der Schwierigkeit, durchsetzungsstarke Unternehmensinteressen kollektiv zu vertreten, Berlin
Behrens, M./Helfen, M. (2009): Innerverbandliche Heterogenität und die Vertretungswirksamkeit deutscher Arbeitgeberverbände, in: Industrielle Beziehungen 16 (1), S. 5-24

Ellguth, P./Kohaut, S. (2013): Tarifbindung und betriebliche Interessenvertretung: Ergebnisse aus dem IAB-Betriebspanel 2012, in: WSI-Mitteilungen 66 (4), S. 281-288, http://www.boeckler.de/wsimit_2013_04_ellguth.pdf Helfen, M. (2006): Wirtschaftsverbände in Deutschland 2006. Zur Leistungsfähigkeit der politischen Organisationen der privaten Wirtschaft, Aachen Helfen, M. (2012): Institutional continuity in German collective bargaining: Do employer associations contribute to stability?, in: Economic and Industrial Democracy 33 (3), S. 485-503

Schmitter, P. C./Streeck, W. (1999): The Organization of Business Interests. Studying the Associative Action of Business in Advanced Industrial Societies: Max-Planck-Institut für Gesellschaftsforschung, MPIfG Discussion Paper 99/1, Köln

Schnabel, C. (2003): Tarifpolitik unter Reformdruck. Benchmarking Deutschland Aktuell, Gütersloh

Schroeder, W. (2010): Geschichte und Funktion der deutschen Arbeitgeberverbände, in: Schroeder, W. Meßels, B. (Hrsg.): Handbuch Arbeitgeber- und Wirtschaftsverbände in Deutschland, Wiesbaden, S. 26-42

Schroeder, W. /Weßels, B. (2010) (Hrsg.): Handbuch Arbeitgeber- und Wirtschaftsverbände in Deutschland, Wiesbaden

Südwestmetall (2012): Professor Hundt: Erfolgsmodell Tarifautonomie nicht in Frage stellen, http://www.suedwestmetall.de/swm/web.nsf/id/li_arbeitgeberbaden-wuerttemberg-zum-jahreswirtschaftsbericht.html

Weitbrecht, H. (2010): Arbeitgeberverbände in der Tarifpolitik und im tarifpolitischen System der Bundesrepublik, in: Schroeder, W./Weßels, B. (Hrsg.): Handbuch Arbeitgeber- und Wirtschaftsverbände in Deutschland, Wiesbaden, S. $316-341$

\section{AUTOR}

MANUEL NICKLICH, Diplom-Soziologe, Wissenschaftlicher Mitarbeiter am Management-Department der Freien Universität Berlin. Arbeitsschwerpunkte: Industrie- und Betriebssoziologie, Organisationssoziologie, industrienahe Dienstleistungen.

manuel.nicklich@fu-berlin.de 